\title{
Flavanone metabolites decrease monocyte adhesion to TNF- $\alpha$-activated endothelial cells by modulating expression of atherosclerosis-related genes
}

\author{
Audrey Chanet ${ }^{1,2}$, Dragan Milenkovic ${ }^{1,2}$, Sylvain Claude ${ }^{1,2}$, Jeanette A. M. Maier ${ }^{3}$, Muhammad Kamran \\ Khan $^{4}$, Njara Rakotomanomana ${ }^{4}$, Svitlana Shinkaruk ${ }^{5}$, Annie M. Bérard ${ }^{6}$, Catherine Bennetau- \\ Pelissero $^{5}$, Andrzej Mazur ${ }^{1,2}$ and Christine Morand ${ }^{1,2 *}$ \\ ${ }^{1}$ INRA, UMR 1019, UNH, CRNH Auvergne, F-63000 Clermont-Ferrand, France \\ ${ }^{2}$ Clermont Université, Université d'Auvergne, Unité de Nutrition Humaine, BP 10448, F-63000, Clermont-Ferrand, France \\ ${ }^{3}$ Dipartimento di Scienze Cliniche Luigi Sacco, Università di Milano, Milano, Italy \\ ${ }^{4}$ INRA, UMR 408, Université d'Avignon, F-84000 Avignon, France \\ ${ }^{5}$ Université de Bordeaux - Bordeaux Sciences Agro, 33175 Gradignan cedex, France \\ ${ }^{6}$ ERU "Facteurs de risque vasculaires", CHU-Université de Bordeaux 2, 146, rue Léo-Saignat, case 49, 33076 Bordeaux, \\ France \\ (Submitted 11 April 2012 - Final revision received 8 November 2012 - Accepted 8 November 2012 - First published online 21 January 2013)
}

\section{Abstract}

Flavanones are found specifically and abundantly in citrus fruits. Their beneficial effect on vascular function is well documented. However, little is known about their cellular and molecular mechanisms of action in vascular cells. The goal of the present study was to identify the impact of flavanone metabolites on endothelial cells and decipher the underlying molecular mechanisms of action. We investigated the impact of naringenin and hesperetin metabolites at $0 \cdot 5,2$ and $10 \mu \mathrm{M}$ on monocyte adhesion to TNF- $\alpha$-activated human umbilical vein endothelial cells (HUVEC) and on gene expression. Except hesperetin-7-glucuronide and naringenin-7-glucuronide (N7G), when present at $2 \mu \mathrm{M}$, flavanone metabolites (hesperetin- $3^{\prime}$-sulphate, hesperetin- $3^{\prime}$-glucuronide and naringenin- $4^{\prime}$-glucuronide (N4' $\mathrm{G}$ ) significantly attenuated monocyte adhesion to TNF- $\alpha$-activated HUVEC. Exposure of both monocytes and HUVEC to N4'G and N7G at $2 \mu \mathrm{M}$ resulted in a higher inhibitory effect on monocyte adhesion. Gene expression analysis, using TaqMan Low-Density Array, revealed that flavanone metabolites modulated the expression of genes involved in atherogenesis, such as those involved in inflammation, cell adhesion and cytoskeletal organisation. In conclusion, physiologically relevant concentrations of flavanone metabolites reduce monocyte adhesion to TNF- $\alpha$-stimulated endothelial cells by affecting the expression of related genes. This provides a potential explanation for the vasculoprotective effects of flavanones.

\section{Key words: Flavanones: Metabolites: Monocyte adhesion: Gene expression: Endothelial cells}

Atherosclerosis, a multifactorial disease of the large arteries and the leading cause of CVD, is characterised by inflammatory and oxidative stress involving chronic dysfunction of the endothelium. This altered function of endothelial cells is associated with both an imbalance in the production of vasodilators/vasoconstrictors and an increase in the expression of pro-inflammatory, chemotactic and adhesion molecules ${ }^{(1,2)}$. These changes facilitate the adhesion of circulating immune cells to the activated endothelium, and their subsequent migration into the arterial wall constitutes early events in the atherogenic process. Once in the intima, monocytes differentiate into activated macrophages, which take up oxidised lipoproteins, leading to the formation of foam cells ${ }^{(3)}$.

A positive association between a high consumption of fruits and vegetables and a reduced risk of CVD has been described $^{(4,5)}$. Among the protective bioactive compounds found in fruits and vegetables, polyphenols seem to be of particular interest. Indeed, epidemiological studies have established an inverse relationship between the intake of flavonoids, a subclass of polyphenols, and mortality from $\mathrm{CVD}^{(6)}$. Animal

Abbreviations: ACTR, actin-related protein; CCL2, chemokine (C-C motif) ligand 2; EZR, ezrin; H3'G, hesperetin-3'-glucuronide; H3'S, hesperetin- $3^{\prime}$ sulphate; H7G, hesperetin-7-glucuronide; H, hesperetin; HUVEC, human umbilical vein endothelial cell; ICAM-1, intercellular adhesion molecule 1; N4'G, naringenin-4'-glucuronide; N7G, naringenin-7-glucuronide; RAC, ras-related C3 botulinum toxin substrate 1; TLDA, TaqMan Low-Density Array; VCAM-1, vascular cell adhesion molecule 1.

*Corresponding author: C. Morand, email christine.morand@clermont.inra.fr 
studies have demonstrated that polyphenols may exert anti-atherogenic effects through anti-oxidative and anti-inflammatory actions that may prevent endothelial dysfunction ${ }^{(7)}$.

Flavanones are a subclass of flavonoids found specifically and abundantly in citrus fruits. Recently, a prospective study demonstrated a positive association between the dietary intake of flavanones and flavanone-rich foods and reduced risk of $\mathrm{CHD}$ and stroke ${ }^{(8,9)}$. In addition, a recent clinical trial established that flavanone supplementation in healthy, middle-aged, moderately overweight men improved postprandial microvascular endothelial reactivity and lowered diastolic blood pressure after 1 month ${ }^{(10)}$. In animal studies, flavanone supplementation has been reported to exert anti-atherogenic properties in various animal models of diet-induced atherosclerosis $^{(11-13)}$. Such effects have been associated with reduced expression of vascular cell adhesion molecule 1 (VCAM-1) and monocyte chemotactic protein-1 in the $\operatorname{aorta}^{(11)}$. We have also shown, in a recent study, that the antiatherogenic effect of flavanones was associated with decreased concentrations of plasma biomarkers of endothelial dysfunction and modulation of expression of genes involved in adhesion and transendothelial migration ${ }^{(13)}$. Furthermore, the few studies that have investigated the effects of flavanones on endothelial cell activity in vitro have reported changes in the levels of cell adhesion molecules and reduced adhesion of monocytes to endothelial cells ${ }^{(14-16)}$. Altogether, these data suggest a beneficial role of flavanone consumption on vascular function.

Cell culture-based studies are important to identify the cellular and molecular mechanisms involved in flavanones' action regarding CVD prevention. However, to date, most of the studies performed have used supraphysiological concentrations of flavanones (aglycone or glycoside forms), which are not present in the circulation after citrus fruit consumption $^{(14,15)}$. Indeed, after ingestion, flavanone glycosides naturally present in citrus fruits are first hydrolysed in the distal part of the intestine by the microflora to release aglycones, which are then absorbed. In the intestinal cells, aglycone forms are subjected to phase II biotransformations (glucuronidation, sulphation and methylation), processes which continue in the liver ${ }^{(17)}$. Consequently, flavanones present in plasma are not native or aglycone forms, but almost exclusively conjugated metabolites. Such metabolites were identified in human subjects as being hesperetin-7-glucuronide $(\mathrm{H} 7 \mathrm{G})$, hesperetin- $3^{\prime}$-sulphate $\left(\mathrm{H} 3^{\prime} \mathrm{S}\right)$, hesperetin-3'-glucuronide $\left(\mathrm{H} 3^{\prime} \mathrm{G}\right)$, naringenin- $4^{\prime}$ glucuronide $\left(\mathrm{N} 4^{\prime} \mathrm{G}\right)$ and naringenin-7-glucuronide (N7G) after consumption of orange and grapefruit juice ${ }^{(18-20)}$. Whether these metabolites at physiologically relevant concentrations have biological potencies remain unknown.

The aim of the present study was to investigate the effect of flavanone metabolites on endothelial cell function, particularly on monocyte adhesion on TNF- $\alpha$-activated endothelial cells. Furthermore, to decipher the underlying molecular mechanisms, the impact of flavanone metabolites on the expression profile of genes and proteins involved in atherosclerosis development have been analysed.

\section{Material and methods}

\section{Materials and chemicals}

Naringenin and hesperetin were purchased from Extrasynthese. Flavanone glucuronides ( $\mathrm{H} 7 \mathrm{G}, \mathrm{H} 3^{\prime} \mathrm{G}$ and $\mathrm{N} 7 \mathrm{G}$ ) and $\mathrm{H} 3^{\prime}$ S were chemically synthesised ${ }^{(20)}$. Chemical structures of naringenin and hesperetin and their main conjugated forms are presented in Supplemental Fig. S1 (available online). Solutions of naringenin, hesperetin as well as of their conjugates were obtained by dissolving them in $50 \%$ ethanol in bidistilled water. TNF- $\alpha$ was obtained from R\&D Systems and was dissolved in $0 \cdot 1 \%$ PBS/bovine serum albumin (Pan Biotech) to obtain a stock solution of $100 \mu \mathrm{g} / \mathrm{ml}$.

\section{Cell culture}

Primary human umbilical vein endothelial cells (HUVEC; Lonza) were used at passage 5 and were cultured in a phenol-red-free endothelial growth medium supplemented with $2 \%$ fetal bovine serum, $0.4 \%$ fibroblast growth factor, $0 \cdot 1 \%$ vascular endothelial growth factor, $0 \cdot 1 \%$ heparin, $0 \cdot 1 \%$ insulin-like growth factor, $0 \cdot 1 \%$ ascorbic acid, $0 \cdot 1 \%$ epidermal growth factor and $0.04 \%$ hydrocortisone (all from Lonza). Experiments were performed in twenty-four-well plates (Becton Dickinson).

A human monocytic cell line (U937) (American Type Culture Collection) was cultured in Roswell Park Memorial Institute medium (Pan Biotech) supplemented with $2 \%$ fetal bovine serum (Sigma). Both cultures were maintained at $37^{\circ} \mathrm{C}$ and $5 \% \mathrm{CO}_{2}$.

\section{Monocyte adhesion assay}

HUVEC were allowed to proliferate until they reached $60-70 \%$ confluence. The medium was then replaced to expose the cells for $24 \mathrm{~h}$ to an experimental medium containing solvent alone (ethanol $0.5 \%$, control wells and vehicle), flavanones or flavanone metabolites $(0 \cdot 5,2$ or $10 \mu \mathrm{M})$. At the end of the $24 \mathrm{~h}$ period, the confluent monolayer was stimulated for $4 \mathrm{~h}$ with $0 \cdot 1 \mathrm{ng} / \mathrm{ml} \mathrm{TNF}-\alpha$ or PBS/bovine serum albumin (0.01\%o, negative control). Following TNF- $\alpha$ stimulation, $50 \mu \mathrm{l}$ of a $5 \times 10^{6} / \mathrm{ml}$ U937 cell suspension were added to each well and cells were further incubated for $1 \mathrm{~h}$. Non-adhering U937 cells were rinsed away using $1 \times$ PBS and the wells were fixed with $0.5 \%$ crystal violet in ethanol (Sigma). The number of attached U937 cells was counted for each well in three random microscopic fields defined by an eyepiece $^{(21)}$. Triplicates for each condition were performed in three independent experiments.

In a second set of experiments, both HUVEC and U937 cells were pre-treated separately for $24 \mathrm{~h}$ with flavanones or flavanone metabolites $(2 \mu \mathrm{M})$. After $24 \mathrm{~h}, 50 \mu \mathrm{l}$ of a suspension of $5 \times 10^{6} / \mathrm{ml}$ U937 cells, pre-exposed to flavanones or their metabolites, were added to the wells containing HUVEC pre-exposed to the same compounds and previously treated for $4 \mathrm{~h}$ with TNF- $\alpha$. The two cell types were co-incubated for $1 \mathrm{~h}$ and the adhesion assay performed as described earlier. 
The experiments were repeated three times in triplicate. The experimental approach is schematically represented in Fig. 1.

\section{RNA extraction and complementary DNA synthesis}

To collect RNA, sub-confluent HUVEC were exposed for $24 \mathrm{~h}$ to a medium containing solvent alone (ethanol $0.5 \%$, control wells), flavanones or flavanone metabolites. The monolayer was stimulated for $5 \mathrm{~h}$ with TNF- $\alpha(0.1 \mathrm{ng} / \mathrm{ml})$ in order to expose the cells to TNF- $\alpha$ for the same time period as in the adhesion assay, where the HUVEC were activated with TNF- $\alpha$ for $4 \mathrm{~h}$ prior to co-incubation with monocytes for a further $1 \mathrm{~h}$. Following this step, the cells were rinsed twice with cold PBS and lysed (Qiagen). Samples were kept at $-80^{\circ} \mathrm{C}$ before RNA extraction.

Total RNA was extracted using QIAGEN ${ }^{\circledR}$ RNeasy ${ }^{\circledR}$ Kits (Qiagen), according to the manufacturer's protocol. The quality of total RNA was monitored in $1 \%$ agarose gel by electrophoresis and RNA quantity was checked on a NanoDrop ND-1000 spectrophotometer (Thermo Scientific). RT of total RNA was performed using a High Capacity cDNA RT kit (Applied Biosystems), each reaction mixture containing $1 \mu \mathrm{g}$ RNA, $1 \times$ RT buffer, 4 mm-deoxyribonucleoside triphosphates (dNTP) mix, $1 \times$ random primers, $50 \mathrm{U}$ RT and $20 \mathrm{U}$ RNase inhibitor in a total volume of $20 \mu \mathrm{l}$. The RT reactions were run under the following conditions: $25^{\circ} \mathrm{C}$ for $10 \mathrm{~min}, 37^{\circ} \mathrm{C}$ for $120 \mathrm{~min}$ and $85^{\circ} \mathrm{C}$ for $5 \mathrm{~s}$.

\section{Real-time quantitative $P C R$}

Impact of flavanones and their metabolites on the expression of genes in HUVEC were performed using the TaqMan LowDensity Array (TLDA) (Applied Biosystems). The TLDA contained primers for ninety-six genes of interest. Genes were chosen according to their role in atherosclerosis development from published articles (Table S1, available online).
A measure of $200 \mathrm{ng}(2 \mu \mathrm{l})$ of complementary DNA was used in each sample and $98 \mu$ l of nuclease-free water together with $100 \mu \mathrm{l} 2 \times$ TaqMan Universal PCR Master Mix (Applied Biosystems) were added for the quantitative real-time PCR measurements. This mixture was divided equally over two sample-loading ports of the TLDA. The arrays were centrifuged once ( $1 \mathrm{~min}, 1300 \mathrm{rpm}$ at room temperature) to equally distribute the sample over the wells. Subsequently, the card was sealed to prevent an exchange between the wells. Quantitative PCR amplification was performed using an Applied Biosystems Prism 7900HT system (Applied Biosystems), with the following thermal cycler conditions: $2 \mathrm{~min}$ at $50^{\circ} \mathrm{C}$ and $10 \mathrm{~min}$ at $94.5^{\circ} \mathrm{C}$, followed by forty cycles of $30 \mathrm{~s}$ at $97^{\circ} \mathrm{C}$ and $30 \mathrm{~s}$ at $59 \cdot 7^{\circ} \mathrm{C}$. Raw data were analysed using Sequence Detection System Software v2.4 (Applied Biosystems). Analyses were performed in triplicate.

\section{Unsupervised modelling}

The clustering analysis was performed on mRNA expression data. Unsupervised modelling was subsequently performed using hierarchical clustering, with the Euclidean distance for calculating the similarity between genes and the Ward's distance for the similarity between conditions using average linkage. Permutmatrix version 1.9.3 (http://www.lirmm.fr/ caraux/ PermutMatrix) was used for hierarchical clustering ${ }^{(22)}$.

\section{Western blot analysis}

For Western blot analysis of proteins, the same experimental conditions as for RNA extraction were used, i.e. the subconfluent HUVEC were exposed for $24 \mathrm{~h}$ to a medium containing solvent alone (ethanol 0.5\%, control wells), flavanones or flavanone metabolites. The monolayer was stimulated for $5 \mathrm{~h}$ with TNF- $\alpha(0 \cdot 1 \mathrm{ng} / \mathrm{ml})$ in order to expose the cell to $\mathrm{TNF}-\alpha$ for the same time period as in adhesion assay, where the HUVEC were activated with TNF- $\alpha$ for $4 \mathrm{~h}$ prior

(a)

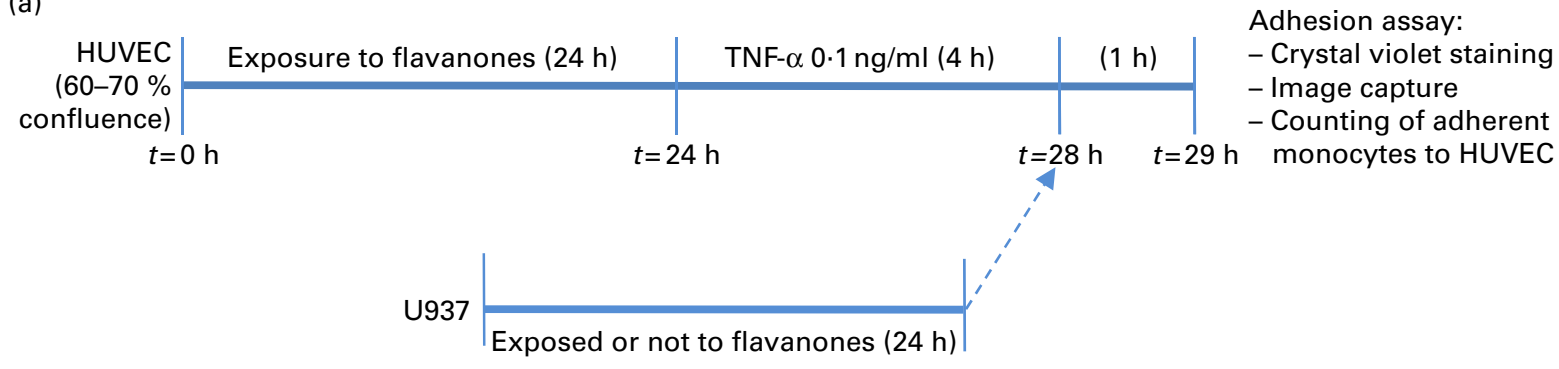

(b)

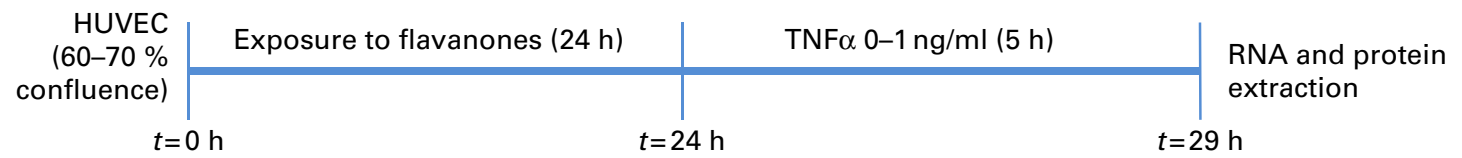

Fig. 1. Schematic representation of in vitro experimental approach. (a) Experimental condition for monocyte adhesion assay. (b) Experimental condition for RNA and protein extraction. HUVEC, human umbilical vein endothelial cells. (A colour version of this figure can be found online at http://www.journals.cambridge.org/bjn) 
to co-incubation with monocytes for further $1 \mathrm{~h}$. Following this step, the cells were rinsed twice with cold PBS and the total proteins were extracted using lysis buffer containing $150 \mathrm{~mm}-\mathrm{NaCl}, 10 \mathrm{~mm}$-Tris-HCl, $1 \mathrm{~mm}$-ethylene glycol tetraacetic acid, $1 \mathrm{~mm}$-EDTA, $100 \mathrm{~mm}$-sodium fluoride, $4 \mathrm{~mm}$ sodium pyrophosphate, $2 \mathrm{~mm}$-sodium orthovanadate, $1 \%$ Triton X100 and 0.5\% IGEPAL CA-630 (all were obtained from Sigma).

After washing with PBS, the cells were lysed using the lysis buffer supplemented with $1 \%$ protease inhibitor cocktail (Sigma) and the lysates were frozen at $-80^{\circ} \mathrm{C}$ overnight after centrifugation at $14000 \mathrm{rpm}$ for $30 \mathrm{~min}$ at $4^{\circ} \mathrm{C}$. Protein concentrations were determined using the BCA Protein Assay Kit (Interchim). A measure of $10 \mu \mathrm{g}$ of total proteins was electrophoretically separated on $8 \%$ polyacrylamide gels and transferred for $1 \mathrm{~h}$ on to nitrocellulose membranes Genetex (Euromedex). The membranes were then blocked for $1 \mathrm{~h}$ with $5 \%$ bovine serum albumin and Tris-buffered saline/0.1\% Tween 20. After washing with Tris-buffered saline/0.1\% Tween 20 , the membranes were incubated with primary antibodies against intercellular adhesion molecule 1 (ICAM-1) (1/2000), VCAM-1 (1/2000), E-Selectin (1/2000), vinculin (1/2000) and $\beta$-actin (1/10000); all the reagents were obtained from Genetex (Euromedex). The membranes were then incubated with secondary fluorescent goat antirabbit and goat anti-mouse IgG $(\mathrm{H}+\mathrm{L})$ antibodies (1/2000) from Genetex (Euromedex). Proteins were visualised by the Odyssey Li-Cor detection system (LI-COR) and the density of the signals was quantified using Odyssey software (LI-COR). Samples were normalised to the housekeeping protein $\beta$-actin. The expression of $\beta$-actin was used as the control for protein loading.

\section{Statistical analyses}

Adhesion assay. Data were analysed by one-way ANOVA, after Bartlett's test of homogeneity of variance and the KolmogorovSmirnov test for Gaussian distribution, followed by Dunnett's post hoc test. A value of $P<0.05$ was considered significant. The software used was GraphPad InStat version 3.06 (GraphPad Software, Inc.).

Gene expression analysis. Relative gene expression levels of each gene were calculated by the comparative $C_{\mathrm{t}}$ method and the results were normalised to actin expression for each sample. Statistical analyses of the normalised gene expression data were performed using Dataassist software (Applied Biosystems; http://products.appliedbiosystems.com/ab/en/US/ adirect/ab?, http://cmd= catNavigate $2 \&$ catID $=606580)$. The software utilises the comparative $C_{\mathrm{T}}\left(\Delta \Delta C_{\mathrm{T}}\right)$ method to calculate quantitative relative gene expression across a large number of genes and samples. The software uses a refined Grubbs' outlier test to remove the outliers among technical replicates, provides a metric to measure control gene stability based on the geNorm algorithm to assist with endogenous control selection and allows the use of single or multiple control genes for data normalisation. $P<0.05$ was taken to imply statistical significance.
Western blot. All experiments were run in three different cell preparations. Results are expressed as means and standard deviations. Statistical analyses were conducted using GraphPad InStat version 3.00 for Windows (GraphPad Software, Inc.). Significance was determined by Student's $t$ test. $P<0.05$ was considered statistically significant.

\section{Results}

Impact of pre-exposure of endothelial cells to flavanone metabolites on monocyte adhesion

Flavanones and their conjugated metabolites at a concentration of $10 \mu \mathrm{m}$ did not significantly influence cell viability compared with flavanone-free control solution (Supplementary data 1 , available online).

As expected, a small number of monocytes adhered to unstimulated HUVEC and treatment with TNF- $\alpha$ significantly increased cell adhesion $(280 \%, P<0.05)$ compared with unstimulated control cells (Fig. 2). Pre-exposure of HUVEC to naringenin and $\mathrm{N} 4^{\prime} \mathrm{G}(2$ and $10 \mu \mathrm{M})$ for $24 \mathrm{~h}$ significantly reduced monocyte adherence to TNF- $\alpha$-activated endothelial cells $(-20 \%)$ (Fig. 2). A reduction in cell adhesion was also observed with N7G, but only at $10 \mu \mathrm{M}$ concentration $(-24 \%)$. Pre-exposure of HUVEC to hesperetin $(\mathrm{H}), \mathrm{H} 3^{\prime} \mathrm{G}$ and $\mathrm{H}^{\prime} \mathrm{S}$ also significantly reduced monocyte adhesion to activated endothelial cells at $2 \mu \mathrm{m}$ concentration $(-23,-21$ and $-14 \%$, respectively), but this effect was no longer observed at a concentration of $10 \mu \mathrm{m}$. Finally, exposure to $\mathrm{H} 7 \mathrm{G}$ did not produce any change in cell adhesion.

\section{Impact of pre-exposure of both endothelial cells and monocytes to flavanone metabolites on cell adhesion}

When both HUVEC and U937 cells were pre-exposed for $24 \mathrm{~h}$ with $N 4^{\prime} G$ and N7G $(2 \mu \mathrm{M})$, the magnitude of the inhibition of cell adhesion was higher compared with the respective conditions with non-exposed monocytes (Fig. 3). For the two aglycone forms as well as for hesperetin metabolites, no differences were observed as compared with non-pretreated U937 cells.

\section{Impact of flavanones and their metabolites on gene expression in TNF- $\alpha$-stimulated endothelial cells}

To evaluate the impact of flavanones and their metabolites on the expression of targeted genes, quantitative PCR was performed using TLDA. Out of ninety-six genes evaluated, eleven were identified as not expressed. Analysis of obtained quantitative PCR raw data by Dataassist software (Applied Biosystems) revealed that the expression of fourteen genes was significantly modified by TNF- $\alpha$. Among these fourteen genes, expression of thirteen was up-regulated and the expression of one gene was down-regulated. Genes modulated by TNF- $\alpha$ are implicated in different cellular processes, such as cell adhesion (ICAM-1 and CDH5 (cadherin 5 , type 2 - vascular endothelium)), inflammation and chemotaxis $(\mathrm{N}-\mathrm{FKB} 1$ and chemokine $(\mathrm{C}-\mathrm{C}$ motif) ligand 2 

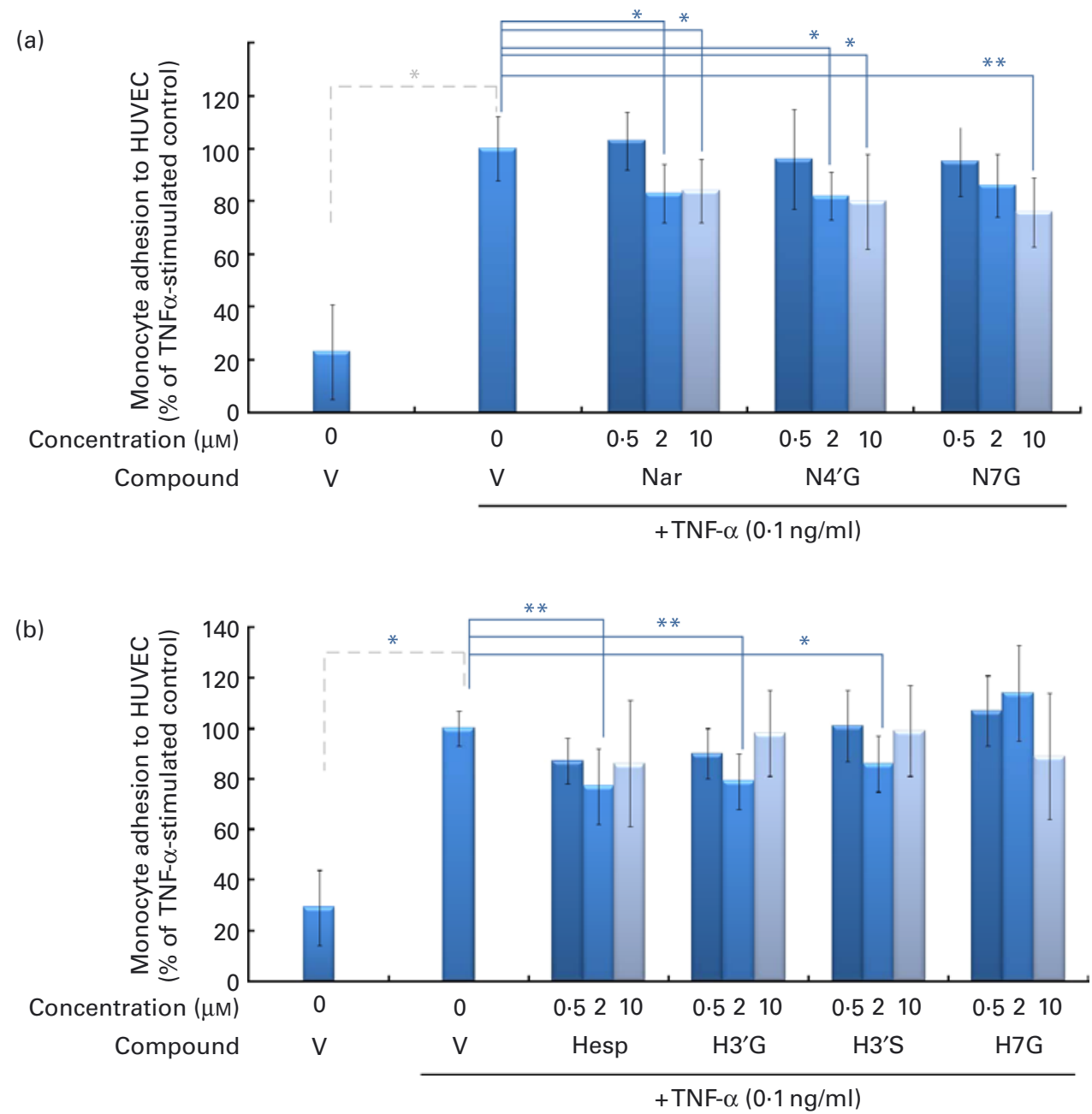

Fig. 2. Effect of incubation of endothelial cells with flavanones on monocyte adhesion. Human umbilical vein endothelial cells (HUVEC) were stimulated with TNF- $\alpha$ for $4 \mathrm{~h}$ after culture with or without (a) naringenin (Nar) and its conjugates (naringenin-4'-glucuronide (N4'G) and naringenin-7-glucuronide (N7G)) or (b) hesperetin (Hesp) and its conjugates (hesperetin-3'-glucuronide $\left(\mathrm{H} 3^{\prime} \mathrm{G}\right)$; hesperetin-3'-sulphate $\left(\mathrm{H} 3^{\prime} \mathrm{S}\right)$ and hesperetin-7-glucuronide $(\mathrm{H} 7 \mathrm{G})$ ) for $24 \mathrm{~h}$. Monocytes were co-incubated for $1 \mathrm{~h}$ and their adhesion to HUVEC counted. Monocyte adhesion is presented as a percentage of U937 to HUVEC. Values are means of three independent experiments, with standard deviations represented by vertical bars. Mean values were significantly different (by oneway ANOVA followed by Dunett's post hoc test): ${ }^{*} P<0.05,{ }^{*} P<0.01$. (A colour version of this figure can be found online at http://www.journals. cambridge.org/bjn)

(CCL2)) or cytoskeleton organisation (actin-related protein (ACTR)2, ezrin (EZR) and RAC-1) (Fig. 4).

This gene expression study also revealed that the $24 \mathrm{~h}$ exposure of HUVEC to flavanones, aglycones or their metabolites at a physiologically relevant concentration $(2 \mu \mathrm{M})$ before activation with TNF- $\alpha$ significantly modulated the expression of genes. $\mathrm{H}_{3}$ 'S modulated the expression of the highest number of genes, as twenty genes have been identified as differentially expressed after $24 \mathrm{~h}$ pre-exposition to this metabolite; $\mathrm{H}, \mathrm{H} 3^{\prime} \mathrm{G}, \mathrm{H} 7 \mathrm{G}$ and $\mathrm{H} 3^{\prime} \mathrm{S}$ modulated the expression of fourteen, seven, six and twenty genes, respectively (Table S2, available online). Naringin and its metabolites, $\mathrm{N} 4^{\prime} \mathrm{G}$ and $\mathrm{N} 7 \mathrm{G}$, modulated the expression of three, nine and four genes, respectively (Table S2, available online). Taken as a whole, modulated genes are implicated in different cellular functions; for example, CD99 molecule (CD99), catenin (cadherin-associated protein), beta $1,88 \mathrm{kDa}$ (CTNNB1), kelch-like ECH-associated protein 1 (KEAP1), moesin (MSN), integrin, beta 1 (ITGB1) and von Willebrand factor (VWF) are involved in cell-cell adhesion; ACTR2, ACTR3, plateletderived growth factor receptor, alpha polypeptide (PDGFRA), p21 protein (Cdc42/Rac)-activated kinase 1 (PAK1), p21 protein (Cdc42/Rac)-activated kinase 4 (PAK4), $E Z R$ and protein phosphatase 1 , regulatory subunit $12 \mathrm{~B}$ $(P P P 1 R 12 B)$ in actin cytoskeleton organisation; ras homolog family member A $(R H O A)$ and cyclin-dependent kinase inhibitor $1(C D K N 1 A)$ in Rho signalling transduction; and CCL2, NFKB1 and mitogen-activated protein kinase 14 (MAPK14) in chemotaxis. As presented earlier, some of the cellular processes affected by flavanone metabolites have also been identified from differentially expressed genes after stimulation of HUVEC with TNF- $\alpha$ compared with non-stimulated cells. Furthermore, most of the aforementioned genes have been identified as regulated by more than one metabolite, while a 

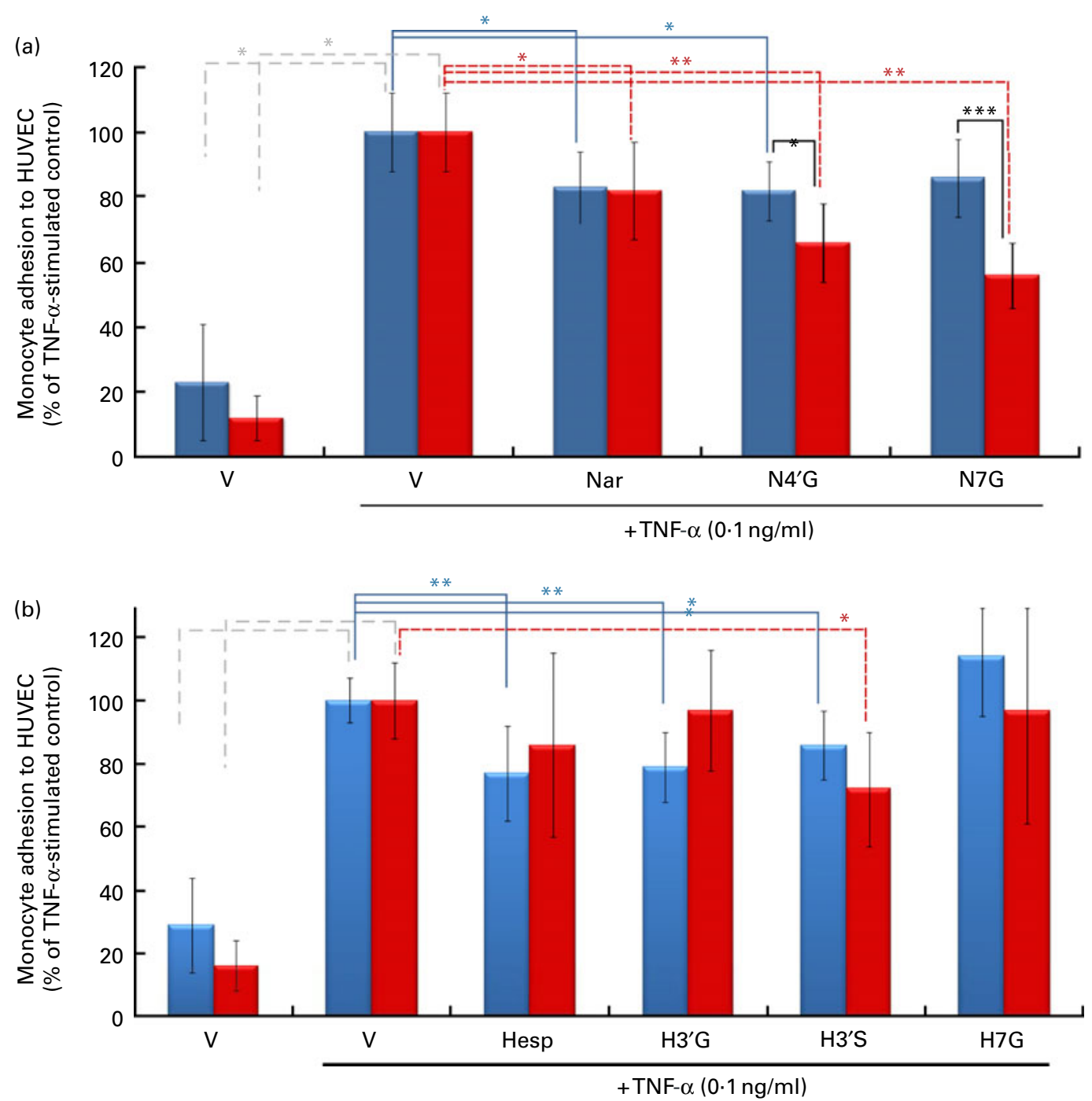

Fig. 3. Impact of pre-exposure of both endothelial cells and monocytes to flavanones on cell adhesion. Human umbilical vein endothelial cells (HUVEC) were stimulated with TNF- $\alpha$ for $4 \mathrm{~h}$ after incubation without or with (a) naringenin (Nar) and its conjugates (naringenin-4'-glucuronide (N4' $\mathrm{G}$ ) and naringenin-7-glucuronide (N7G)) or (b) hesperetin (Hesp) and its conjugates (hesperetin- $3^{\prime}$-glucuronide $\left(\mathrm{H} 3^{\prime} \mathrm{G}\right)$; hesperetin- $3^{\prime}$-sulphate $\left(\mathrm{H}^{\prime} \mathrm{S}\right)$ and hesperetin-7-glucuronide $(\mathrm{H} 7 \mathrm{G})$ ) at $2 \mu \mathrm{M}$ for $24 \mathrm{~h}$. Prior to the adhesion assay, monocytes (U937) were pre-incubated or not with (a) Nar and its conjugates or with (b) Hesp and its conjugates at $2 \mu \mathrm{M}$ for $24 \mathrm{~h}$, co-incubated for $1 \mathrm{~h}$ and their adhesion to HUVEC investigated. Monocyte adhesion is presented as a percentage of U937 to HUVEC. Values are means of three independent experiments, with standard deviations represented by vertical bars. Mean values were significantly different (by one-way ANOVA followed

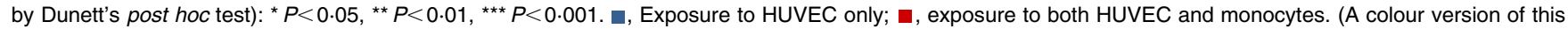
figure can be found online at http://www.journals.cambridge.org/bjn)

few of them, such as KEAP1 or CCL2, have been identified as modulated by only one of the studied metabolites.

The obtained expression profiles of the studied genes have been clustered using the hierarchical unsupervised method using PermutMatrix software. In this cluster, vertical lines represent different experimental conditions, while horizontal lines present the studied genes (Fig. 4). The cluster revealed two distinct groups, one corresponding to the expression profile of cells stimulated with $\mathrm{TNF}-\alpha$, noted $\mathrm{A}$, and the second one containing the expression profiles of cells preexposed to flavanones and then stimulated with TNF- $\alpha$, noted B. This cluster revealed that TNF- $\alpha$ stimulation of HUVEC induced up-regulation of most of the genes, while pre-exposure of HUVEC to flavanones results in a global down-regulation of gene expression.
Effect of flavanones and their metabolites on cell adhesion protein expression levels in TNF- $\alpha$-stimulated endothelial cells

Together with gene expression analysis, protein expression was also analysed for flavanones and their metabolites that significantly reduced monocyte adhesion to HUVEC at $2 \mu \mathrm{M}$ concentration $\left(\mathrm{H}, \mathrm{H} 3^{\prime} \mathrm{G}, \mathrm{H} 3^{\prime} \mathrm{S}\right.$, naringenin and $\left.\mathrm{N} 4^{\prime} \mathrm{G}\right)$. Western blot analysis was performed for cell adhesion molecule proteins (ICAM1, VCAM1 and E-selectin) and cytoskeleton organisation protein (vinculin). The treatment of HUVEC with $\mathrm{H}, \mathrm{H}^{\prime}{ }^{\prime} \mathrm{S}$ and naringenin $(2 \mu \mathrm{M})$ for $24 \mathrm{~h}$ prior to exposure to TNF- $\alpha$ induced a significantly decreased protein level of vinculin (Fig. 5), whereas no impact of the tested metabolites was observed on cell adhesion protein levels (Fig. S2, available online). 


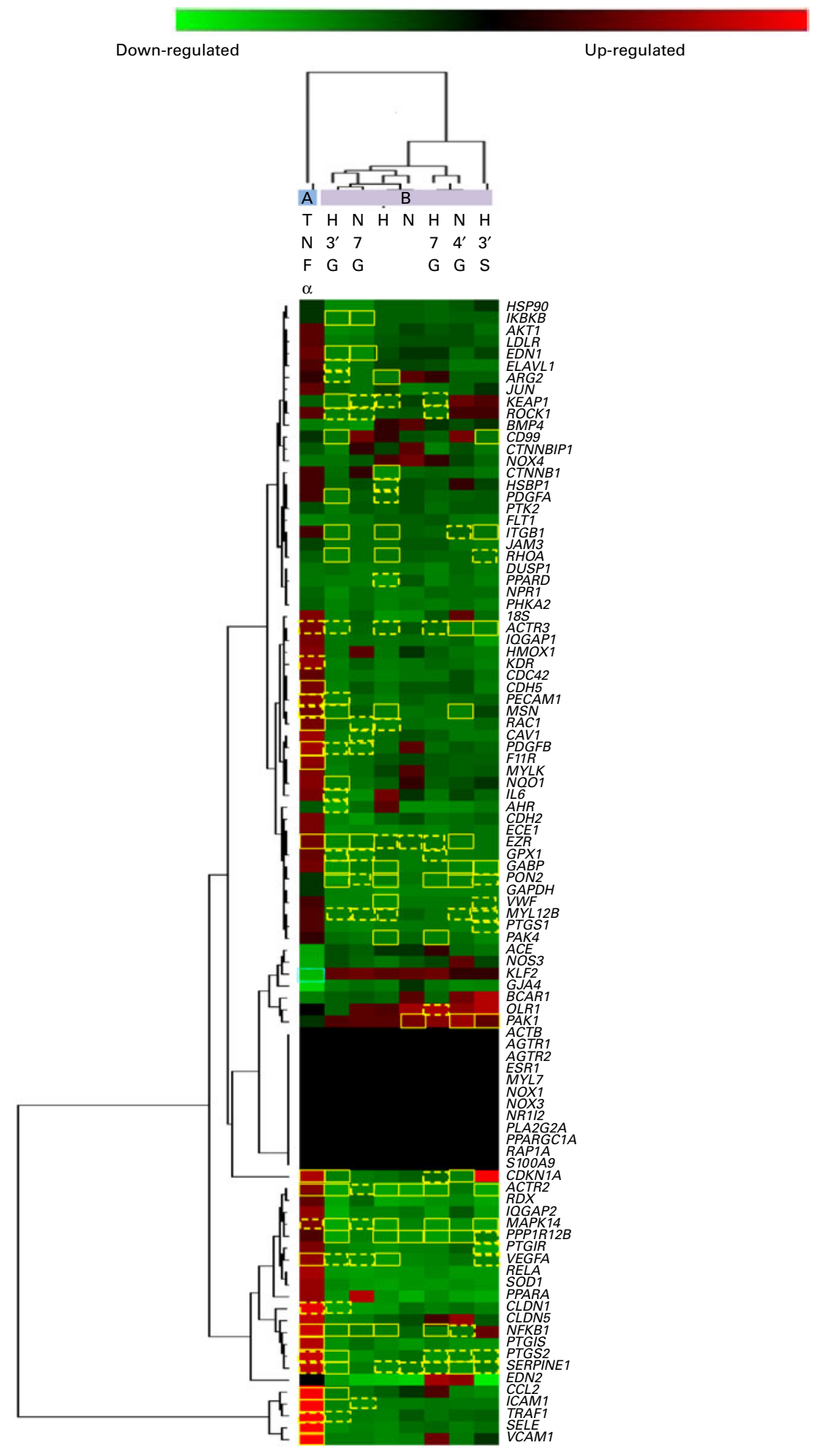

Fig. 4. Two-dimensional hierarchical clustering analysis of mRNA profiles in human umbilical vein endothelial cells (HUVEC) under different conditions (TNF- $\alpha v$. control, TNF- $\alpha$-stimulated HUVEC treated with flavanones $v$. TNF- $\alpha$ alone-stimulated HUVEC). Flavanones, aglycones (hesperetin $(H)$ and naringenin $(N))$ and metabolites (hesperetin-3'-glucuronide $\left(H 3^{\prime} G\right)$, hesperetin-3'-sulphate $\left(H 3^{\prime} S\right)$, hesperetin-7-glucuronide $(H 7 G)$, naringenin- $4^{\prime}$-glucuronide $\left(N 4^{\prime} G\right)$ and naringenin-7glucuronide (N7G)) were used at $2 \mu \mathrm{M}$ concentration. mRNA are represented on the vertical axis, while different tested conditions are plotted on the horizontal axis. Red or green colours indicate up-regulation and down-regulation, respectively. Results are from three independent experiments. Significance was determined by the comparative $C_{\mathrm{T}}\left(\Delta \Delta C_{\mathrm{T}}\right)$ method. Yellow squares $(\square): P<0.05$, blue dotted squares $(,, \mathrm{m}): P<0 \cdot 1$. See Appendix 1 and Table $S 1$ (available online) for a list of names and abbreviations. (A colour version of this figure can be found online at http://www.journals.cambridge.org/bjn) 

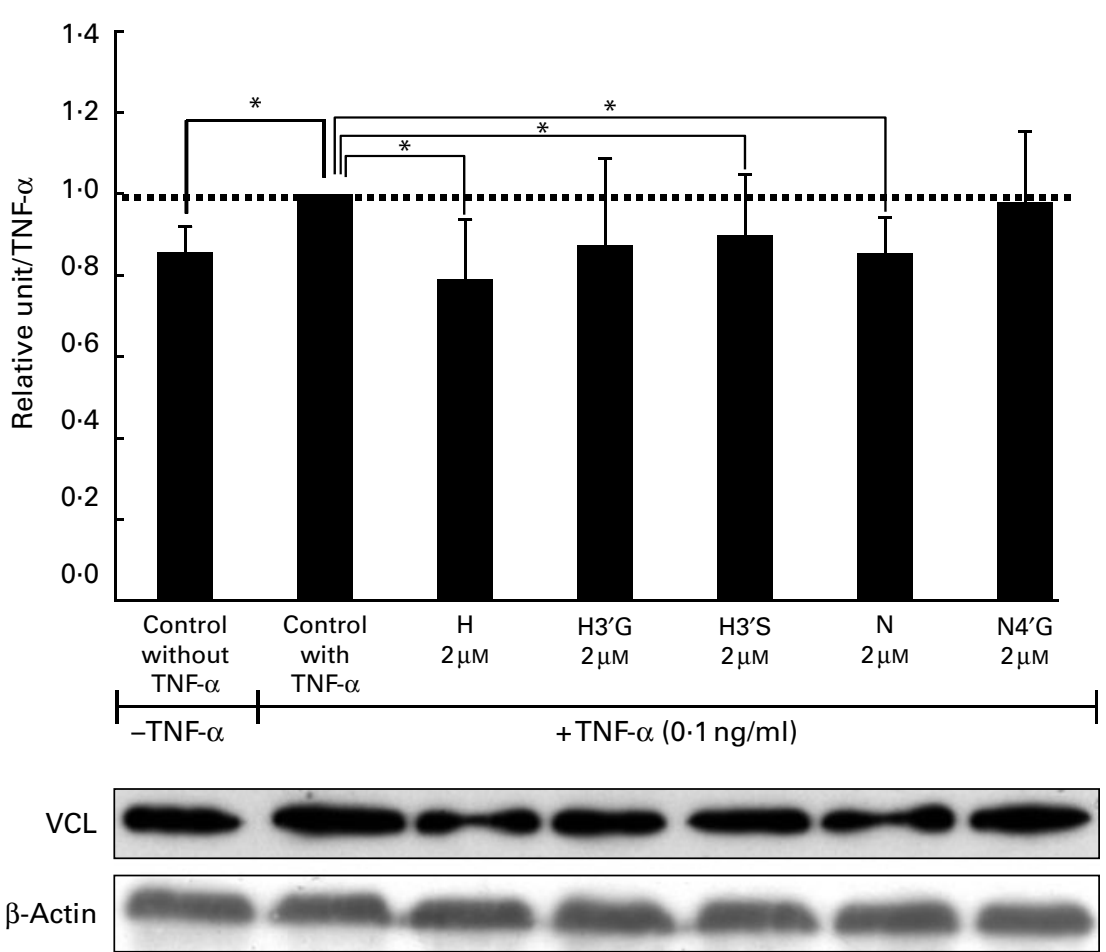

Fig. 5. Effect of flavanones and their metabolites on vinculin (VCL) protein expression in human umbilical vein endothelial cells (HUVEC). HUVEC were pre-treated with hesperetin $(H)$, hesperetin-3'-glucuronide $\left(H 3^{\prime} G\right)$, hesperetin- $3^{\prime}$-sulphate $\left(H 3^{\prime} S\right)$, naringenin $(N)$ or naringenin- $4^{\prime}$-glucuronide $\left(N 4^{\prime} G\right)$ for $24 \mathrm{~h}$ followed by induction with TNF- $\alpha$. Expression of VCL protein in cells was evaluated by immunoblotting. Values are means, with their standard errors represented by vertical bars, $n$ 3. *Mean value was significantly different from that of TNF- $\alpha$ control without flavanone pre-treatment $(P<0 \cdot 05)$.

\section{Discussion}

In a previous in vivo study, we demonstrated that flavanones consumed at a nutritional dose reduced lesion development in a mouse model of diet-induced atherosclerosis ${ }^{(23)}$. Transcriptomic data analysis from aorta revealed that this effect might be mediated by flavanone-induced altered expression of genes that govern monocyte adhesion to endothelial cells and their subsequent trans-endothelial migration into the intima of aorta. Thus, to gain further insights into the role of flavanones on cardiovascular protection, the present study aimed to investigate the direct effects of circulating flavanone metabolites on endothelial cell function. Experimental conditions were set in order to reproduce the vascular environment observed after the consumption of flavanonerich foods. From this perspective, flavanone metabolites that had been identified in human plasma after the consumption of orange and grapefruit juice were used. These metabolites were employed at physiologically relevant concentrations and were incubated with endothelial cells for $24 \mathrm{~h}$, which corresponds to the time required for flavanones to be excreted following their ingestion ${ }^{(23)}$. Furthermore, HUVEC were used as a model of human endothelial cells and were exposed to inflammatory stress to mimic a pro-atherogenic environment. To induce inflammatory stress, TNF- $\alpha$, a pro-inflammatory cytokine capable of inducing endothelial dysfunction and whose circulating concentrations have been identified as elevated in plasma of patients at risk of CVD, was chosen ${ }^{(24)}$. The present study showed that, except $\mathrm{H} 7 \mathrm{G}$ and $\mathrm{N} 7 \mathrm{G}$, flavanone metabolites were effective at $2 \mu \mathrm{m}$ to attenuate monocyte adhesion to TNF- $\alpha$-activated endothelial cells, the first step of atherogenesis. This concentration has been reported in human plasma following grapefruit or orange consumption $^{(25,26)}$ and can be therefore considered as nutritionally achievable. In the present experimental conditions, we failed to observe a dose-dependent effect of flavanone metabolites on cell adhesion. A similar observation has been previously reported for other flavonoids, both in endothelial and in immune cells ${ }^{(27,28)}$. Data from the present study suggest that metabolites could be as potent as their aglycones in preventing monocyte adhesion to activated HUVEC. Furthermore, it is noteworthy that flavanone metabolites conjugated on the $\mathrm{B}$ ring seem to be more efficient in reducing cell adhesion than those substituted on the A ring. This suggests that the biological potency of metabolites depends on the position of conjugation rather than on the nature of the substituent (sulphate or glucuronide). Overall, the ability of flavanone metabolites to decrease monocyte adhesion to activated endothelial cells may be one of the mechanisms underlying the anti-atherogenic activity of flavanones in vivo ${ }^{(10-13)}$.

When present in blood circulation, flavanone metabolites may interact with endothelial cells as well as with immune cells, such as monocytes. Consequently, to fit as closely as possible with physiological conditions, it has been examined to what extent the pre-exposure of both endothelial cells and monocytes to flavanone metabolites may affect the cell adhesion process. The obtained data showed that exposure of monocytes to $N 4^{\prime} \mathrm{G}$ and $\mathrm{N} 7 \mathrm{G}$ potentiated the inhibitory 
effect of flavanones on monocyte adhesion to HUVEC when compared with a condition in which only HUVEC were preexposed to metabolites (Fig. 3). This finding revealed that circulating monocytes also constitute cellular targets for naringenin metabolites, which affect the ability of monocytes to interact with endothelial cells. In agreement, we have previously shown in human subjects that hesperidin supplementation modulated gene expression in leucocytes, in particular genes involved in chemotaxis, adhesion or infiltration $^{(29)}$.

Gene expression analysis in endothelial cells using TaqMan low-density arrays suggested that flavanones could affect endothelial function through transcriptional regulation of gene expression. Genes whose expression was identified as modulated by TNF- $\alpha$ are involved in different cellular processes, such as cell adhesion (ICAM-1 and CDH5), inflammation and chemotaxis (NFKB1 and CCL2) or cytoskeleton organisation (ACTR2, EZR and RAC-1). This observed effect of TNF- $\alpha$ on gene expression has been previously reported $^{(30)}$. Exposure of endothelial cells to flavanones, aglycones or to their metabolites resulted in changes in expression of genes. Most of the modulated genes are involved in various cellular processes, including cell adhesion, cytoskeleton organisation, inflammation and chemotaxis, all being related to atherogenesis. Clustering of gene expression profiles revealed a specific pattern of gene expression in HUVEC activated by TNF- $\alpha$. Interestingly, genes whose expression was up-regulated by TNF- $\alpha$ seemed to be down-regulated by flavanones and vice versa (Fig. 4). This observation revealed the potency of flavanone metabolites, at physiologically relevant concentrations, to counteract the effect induced by an inflammatory stimulus (TNF- $\alpha$ ). As a result, genes related to inflammation could be the molecular targets associated with the reduction of monocyte adhesion to endothelial cells exposed to flavanone metabolites.

The inflammation process plays a critical role in atherosclerosis development through the recruitment of immune cells to the inflammatory site, particularly by triggering the synthesis of adhesion and chemotactic molecules. The present study showed that exposure of endothelial cells to flavanones, aglycones or metabolites induced an anti-inflammatory profile, as revealed by a down-regulation of expression of genes coding for inflammatory mediators, genes whose expression has been induced by TNF- $\alpha$. NFKB1 gene expression was down-regulated by $\mathrm{H} 3^{\prime} \mathrm{S}, \mathrm{N} 7 \mathrm{G}, \mathrm{H}$ and $\mathrm{H} 7 \mathrm{G}$ compared with the TNF- $\alpha$-stimulated control. NFKB1 encodes for a NF- $\mathrm{KB}$ subunit, the well-known transcription factor involved in inflammation and activated by TNF- $\alpha^{(31)}$. In agreement with the decreased expression of NFKB1 gene in response to flavanones, a trend towards the down-regulation of some of its target genes, like CCL2 (also called monocyte chemotactic protein-1 and involved in the chemotaxy of monocytes to endothelial cells) or Traf1 (one member of the TNF- $\alpha$ receptor $)^{(32,33)}$ is observed. In addition, pre-exposure of endothelial cells to flavanones, aglycones or metabolites also affected the expression of genes coding for adhesion molecules, which constitute other targets of NF-кB. For example, flavanones decreased expression of genes coding for adhesion molecules like CD99 or ITGB1 and tended to reduce the expression of genes coding for E-selectin, ICAM-1 and VCAM-1 when compared with the TNF- $\alpha$-stimulated control. These adhesion molecules participate in the rolling and firm adhesion of leucocytes to activated endothelium, and their down-regulation result in the impairment of monocyte adhesion to endothelial cells ${ }^{(14)}$. Overall, these results suggest that flavanones could inhibit inflammatory process through changes in the expression of inflammation-related genes. These genomic effects of flavanones could be, at least partially, mediated by their capacity to affect the expression of the NF- $\mathrm{BB}$ transcription factor. This hypothesis is supported by other studies which have previously shown the ability of flavanones to suppress NF- $\mathrm{B}$ activation and related gene expression in rat kidneys or activated macrophages ${ }^{(34-36)}$.

Leucocyte adhesion on activated endothelium promotes the remodelling of the apical endothelial plasma membrane into actin-rich projections that surround adherent leucocytes, known as a docking structure ${ }^{(37)}$. These transmigratory cups participate in firm adhesion of monocytes to the endothelial cells and serve as directional guides for leucocytes to cross the endothelium ${ }^{(38)}$. These features of the actin cytoskeleton are also regulated by a cohort of actin-associated cytoskeletal proteins, such as talin, vinculin or cofilin. The complex constituted by ICAM-1 and VCAM-1 associated to ERM proteins (EZR, radixin and moesin) initiates intracellular signalling, such as activation of the small GTPase pathway ${ }^{(37,39)}$. Interestingly, TLDA analysis revealed that, when compared with the TNF- $\alpha$-stimulated control, pre-exposure of endothelial cells to flavanones also down-regulated the expression of genes coding for ERM proteins: EZR, moesin and small GTPase members. In response to flavanones, the down-regulation of EZR reached significance for $H 3^{\prime} S, N 7 G$ and $N 4^{\prime} G$ and that of moesin for $\mathrm{H}^{\prime} \mathrm{S}, \mathrm{H}$ and $\mathrm{N}^{\prime} \mathrm{G}$. Pre-exposure of HUVEC to flavanones also resulted in a decreased expression of RAC1, a member of the rho family of small GTPase and whose inhibition reduces leucocyte extravasation ${ }^{(37,39,40)}$. RAC signalling is known to lead to actin polymerisation through the activation of actin-related protein (ARP) $2 / 3$ complex $^{(41)}$, which in turn allows transmigration ${ }^{(42)}$. Interestingly, the present study also showed that most of the studied flavanone compounds significantly down-regulated the expression of both ARP2 and ARP3 genes coding for ACTR2 and ACTR3 proteins, which are major constituents of the ARP $2 / 3$ complex. Furthermore, gene expression analysis revealed differentially expressed genes involved in cytoskeleton organisation. Using Western blot analysis, we examined expression of vinculin, a protein involved in cytoskeleton organisation and remodelling. This analysis revealed a decrease in vinculin protein level in endothelial cells pre-exposed to flavanone metabolites (Fig. 5). Overall, the reduced gene expression of actin cytoskeleton, docking structure components, cytoskeleton organisation or their regulators by flavanones (aglycones and their metabolites) might result in decreased transmigratory cups formation and consequently lead to a lower monocyte adhesion to endothelial cells and transendothelial migration. 
In conclusion, the present study reveals that at physiologically achievable concentrations, the flavanone metabolites present in human plasma following citrus fruit consumption retain the ability to decrease monocyte adhesion to TNF- $\alpha$-stimulated endothelial cells. These metabolites also present potency to modulate expression of genes, particularly those coding for cell adhesion molecules, chemokines and cytoskeleton components that are involved in monocyte adhesion and recruitment in the vascular wall. The observed modulation of gene expression at the transcriptional level by flavanones might be related to the decreased adhesion of monocytes to endothelial cells. These data give an insight into potential cellular and molecular mechanisms responsible for the athero-protective effects of dietary flavanones reported in vivo.

\section{Supplementary material}

To view supplementary material for this article, please visit http://dx.doi.org/10.1017/S0007114512005454

\section{Acknowledgements}

We would like to thank Christiane Deval for TLDA experiments and Nicolas Gerard for Western blot analysis. We thank UNIJUS, the French association of fruit juice producers, who followed and communicated about this research programme. A. C. contributed to the conception and design of the study, acquisition, analysis and interpretation of data; writing the article and the final approval of the manuscript. D. M. contributed to the conception and design of the study, acquisition, analysis and interpretation of gene expression data; writing the article and the final approval of the manuscript. S. C. contributed to the Western blot analyses, writing the article and the final approval of the manuscript. J. A. M. M. contributed to the conception of the study, analysis and interpretation of cell adhesion data; writing the article and the final approval of the manuscript. M. K. K. contributed to the conception of the study, production and analysis of metabolites and the final approval of the manuscript. N. R. contribution to the conception of the study, production and analysis of metabolites and the final approval of the manuscript. S. S. contributed to the conception of the study, production and analysis of metabolites and the final approval of the manuscript. A. M. B. contributed to the development of the study protocol, revising of the article and the final approval of the manuscript. C. B.-P. contributed to the development of the study protocol, critical revising of the article and the final approval of the manuscript. A. M. contributed to the development of the study protocol, statistical analysis of the data and the final approval of the manuscript. C. M. contributed to the conception and design of the study, analysis and interpretation of data; writing the article and the final approval of the version to be published. The authors do not have financial/commercial conflict of interest. This project has received funding from the French National Research Agency in the context of the National Program for Research on Food and Nutrition (PNRA).

\section{References}

1. Widlansky ME, Gokce N, Keaney JF Jr, et al. (2003) The clinical implications of endothelial dysfunction. J Am Coll Cardiol 42, 1149-1160.

2. Sima AV, Stancu CS \& Simionescu M (2009) Vascular endothelium in atherosclerosis. Cell Tissue Res 335, 191-203.

3. Lusis AJ (2000) Atherosclerosis. Nature 407, 233-241.

4. Dauchet L, Amouyel P \& Dallongeville J (2005) Fruit and vegetable consumption and risk of stroke - a meta-analysis of cohort studies. Neurology 65, 1193-1197.

5. Dauchet L, Amouyel P, Hercberg S, et al. (2006) Fruit and vegetable consumption and risk of coronary heart disease: a meta-analysis of cohort studies. J Nutr 136, 2588-2593.

6. Arts ICW \& Hollman PCH (2005) Polyphenols and disease risk in epidemiologic studies. Am J Clin Nutr 81, 317s-325s.

7. Auclair S, Milenkovic D, Besson C, et al. (2009) Catechin reduces atherosclerotic lesion development in apo E-deficient mice: a transcriptomic study. Atherosclerosis 204, e21-e27.

8. Mink PJ, Scrafford CG, Barraj LM, et al. (2007) Flavonoid intake and cardiovascular disease mortality: a prospective study in postmenopausal women. Am J Clin Nutr 85, 895-909.

9. Cassidy A, Rimm EB, O'Reilly EJ, et al. (2012) Dietary flavonoids and risk of stroke in women. Stroke 43, 946-951.

10. Morand C, Dubray C, Milenkovic D, et al. (2011) Hesperidin contributes to the vascular protective effects of orange juice: a randomized crossover study in healthy volunteers. Am J Clin Nutr 93, 73-80.

11. Lee CH, Jeong TS, Choi YK, et al. (2001) Anti-atherogenic effect of citrus flavonoids, naringin and naringenin, associated with hepatic ACAT and aortic VCAM-1 and MCP-1 in high cholesterol-fed rabbits. Biochem Biophys Res Commun 284, 681-688.

12. Mulvihill EE, Assini JM, Sutherland BG, et al. (2010) Naringenin decreases progression of atherosclerosis by improving dyslipidemia in high-fat-fed low-density lipoprotein receptor-null mice. Arterioscler Thromb Vasc Biol 30, 742-748.

13. Chanet A, Milenkovic D, Deval C, et al. (2012) Naringin, the major grapefruit flavonoid, specifically affects atherosclerosis development in diet-induced hypercholesterolemia in mice. J Nutr Biochem 23, 469-477.

14. Choi JS, Choi YJ, Park SH, et al. (2004) Flavones mitigate tumor necrosis factor-alpha-induced adhesion molecule upregulation in cultured human endothelial cells: role of nuclear factor-kappa B. J Nutr 134, 1013-1019.

15. Nizamutdinova IT, Jeong JJ, Xu GH, et al. (2008) Hesperidin, hesperidin methyl chalone and phellopterin from Poncirus trifoliata (Rutaceae) differentially regulate the expression of adhesion molecules in tumor necrosis factor-alphastimulated human umbilical vein endothelial cells. Int Immunopharmacol 8, 670-678.

16. Rizza S, Muniyappa R, Iantorno M, et al. (2011) Citrus polyphenol hesperidin stimulates production of nitric oxide in endothelial cells while improving endothelial function and reducing inflammatory markers in patients with metabolic syndrome. J Clin Endocrinol Metab 96, E782-E792.

17. Manach C, Scalbert A \& Morand C (2004) Polyphenols: food sources and bioavailability. Am J Clin Nutr 79, 727-747.

18. Vallejo F, Larrosa M, Escudero E, et al. (2010) Concentration and solubility of flavanones in orange beverages affect their bioavailability in humans. J Agric Food Chem 58, 6516-6524.

19. Brett GM, Hollands W, Needs PW, et al. (2009) Absorption, metabolism and excretion of flavanones from single portions of orange fruit and juice and effects of anthropometric 
variables and contraceptive pill use on flavanone excretion. Br J Nutr 101, 664-675.

20. Khan MK, Rakotomanomana N, Loonis M, et al. (2010) Chemical synthesis of citrus flavanone glucuronides. J Agric Food Chem 58, 8437-8443.

21. Maier JA, Malpuech-Brugere C, Zimowska W, et al. (2004) Low magnesium promotes endothelial cell dysfunction: implications for atherosclerosis, inflammation and thrombosis. Biochim Biophys Acta 1689, 13-21.

22. Caraux G \& Pinloche S (2005) PermutMatrix: a graphical environment to arrange gene expression profiles in optimal linear order. Bioinformatics 21, 1280-1281.

23. Chanet A, Milenkovic D, Manach C, et al. (2012) Citrus flavanones: what is their role in cardiovascular protection? J Agric Food Chem 60, 8809-8822.

24. Zhang H, Park Y, Wu J, et al. (2009) Source role of TNFalpha in vascular dysfunction. Clin Sci (Lond) 116, 219-230.

25. Erlund I, Meririnne E, Alfthan G, et al. (2001) Plasma kinetics and urinary excretion of the flavanones naringenin and hesperetin in humans after ingestion of orange juice and grapefruit juice. J Nutr 131, 235-241.

26. Erlund I, Silaste ML, Alfthan G, et al. (2002) Plasma concentrations of the flavonoids hesperetin, naringenin and quercetin in human subjects following their habitual diets, and diets high or low in fruit and vegetables. Eur J Clin Nutr 56, $891-898$.

27. Tribolo S, Lodi F, Connor C, et al. (2008) Comparative effects of quercetin and its predominant human metabolites on adhesion molecule expression in activated human vascular endothelial cells. Atherosclerosis 197, 50-56.

28. de Pascual-Teresa S, Johnston KL, DuPont MS, et al. (2004) Quercetin metabolites downregulate cyclooxygenase-2 transcription in human lymphocytes ex vivo but not in vivo. J Nutr 134, 552-557.

29. Milenkovic D, Deval C, Dubray C, et al. (2011) Hesperidin displays relevant role in the nutrigenomic effect of orange juice on blood leukocytes in human volunteers: a randomized controlled cross-over study. PLoS One 6, e26669.

30. Perrot-Applanat M, Vacher S, Toullec A, et al. (2011) Similar NF-kB gene signatures in TNF- $\alpha$ treated human endothelial cells and breast tumor biopsies. PLoS One 6, e21589.
31. Monaco C \& Paleolog E (2004) Nuclear factor kappaB: a potential therapeutic target in atherosclerosis and thrombosis. Cardiovasc Res 61, 671-682.

32. Toborek M \& Kaiser S (1999) Endothelial cell functions. Relationship to atherogenesis. Basic Res Cardiol 94, 295-314.

33. Wang CY, Mayo MW, Korneluk RG, et al. (1998) NF-kappaB antiapoptosis: induction of TRAF1 and TRAF2 and c-IAP1 and C-IAP2 to suppress caspase- 8 activation. Science $\mathbf{2 8 1}$, $1680-1683$.

34. Kim JY, Jung KJ, Choi JS, et al. (2006) Modulation of the agerelated nuclear factor-kappaB (NF-kappaB) pathway by hesperetin. Aging Cell 5, 401-411.

35. Hamalainen M, Nieminen R, Vuorela P, et al. (2007) Antiinflammatory effects of flavonoids: genistein, kaempferol, quercetin, and daidzein inhibit STAT-1 and NF-kappaB activations, whereas flavone, isorhamnetin, naringenin, and pelargonidin inhibit only NF-kappaB activation along with their inhibitory effect on iNOS expression and NO production in activated macrophages. Mediators Inflamm 2007, 45673.

36. Tsai SH, Lin-Shiau SY \& Lin JK (1999) Suppression of nitric oxide synthase and the down-regulation of the activation of NFkappaB in macrophages by resveratrol. BrJ Pharmacol 126, 673-680.

37. Hordijk PL (2006) Endothelial signalling events during leukocyte transmigration. FEBS J 273, 4408-4415.

38. Wittchen ES (2009) Endothelial signaling in paracellular and transcellular leukocyte transmigration. Front Biosci 14, $2522-2545$.

39. van Buul JD \& Hordijk PL (2004) Signaling in leukocyte transendothelial migration. Arterioscler Thromb Vasc Biol 24, 824-833.

40. Millan J \& Ridley AJ (2005) Rho GTPases and leucocyteinduced endothelial remodelling. Biochem J 385, 329-337.

41. Eden S, Rohatgi R, Podtelejnikov AV, et al. (2002) Mechanism of regulation of WAVE1-induced actin nucleation by Rac1 and Nck. Nature 418, 790-793.

42. Yang L, Kowalski JR, Yacono P, et al. (2006) Endothelial cell cortactin coordinates intercellular adhesion molecule-1 clustering and actin cytoskeleton remodeling during polymorphonuclear leukocyte adhesion and transmigration. J Immunol 177, 6440-6449. 
Appendix 1. Gene abbreviations and names

\begin{tabular}{|c|c|c|c|}
\hline $18 S$ & RNA, $18 S$ ribosomal 0 & NQO1 & $\mathrm{NAD}(\mathrm{P}) \mathrm{H}$ dehydrogenase, quinone 1 \\
\hline$A C E$ & Angiotensin I converting enzyme & \multirow[t]{2}{*}{ NRII2 } & Nuclear receptor subfamily 1 \\
\hline ACTB & Actin, beta & & group I, member 1 \\
\hline ACTR2 & Actin-related protein 2 & \multirow[t]{2}{*}{ OLR1 } & Oxidized low density lipoprotein \\
\hline ACTR3 & Actin-related protein 3 & & (lectin-like) receptor 1 \\
\hline AGTR1 & Angiotensin II receptor, type 1 & PAK1 & p21 protein (Cdc42/Rac)-activated kinase 1 \\
\hline AGTR2 & Angiotensin II receptor, type 2 & PAK4 & p21 protein (Cdc42/Rac)-activated kinase 4 \\
\hline$A H R$ & Aryl hydrocarbon receptor & \multirow[t]{2}{*}{ PDGFA } & Platelet-derived growth factor alpha \\
\hline$A K T 1$ & v-akt murine thymoma viral & & polypeptide \\
\hline & oncogene homolog 1 & \multirow[t]{2}{*}{$P D G F B$} & Platelet-derived growth factor beta \\
\hline ARG2 & Arginase, type II & & polypeptide \\
\hline$B C A R 1$ & $\begin{array}{l}\text { Breast cancer anti-estrogen resistance } \\
\text { protein } 1\end{array}$ & $\begin{array}{l}\text { PECAM1 } \\
\text { PHKA2 }\end{array}$ & $\begin{array}{l}\text { Platelet/endothelial cell adhesion molecule } 1 \\
\text { Phosphorylase kinase, alpha } 2\end{array}$ \\
\hline BMP4 & Bone morphogenetic protein 4 & PLA2G2A & Phospholipase A2, group IIA \\
\hline CAV1 & Caveolin 1 & PON2 & Paraoxonase 2 \\
\hline CCL2 & Hemokine (C-C motif) ligand 2 & PPARA & Peroxisome proliferator-activated receptor alpha \\
\hline CD99 & CD99 molecule & PPARD & Peroxisome proliferator-activated receptor delta \\
\hline$C D C 42$ & Cell division control protein 42 homolog & \multirow[t]{2}{*}{ PPARGC1A } & \multirow{2}{*}{$\begin{array}{l}\text { Peroxisome proliferator-activated receptor gamma, } \\
\text { coactivator } 1 \text { alpha }\end{array}$} \\
\hline $\mathrm{CDH} 2$ & Cadherin-2 & & \\
\hline $\mathrm{CDH} 5$ & Cadherin-5 & \multirow[t]{2}{*}{$P P P 1 R 12 B$} & \multirow{2}{*}{$\begin{array}{l}\text { Protein phosphatase } 1 \text {, regulatory } \\
\text { subunit } 12 \mathrm{~B}\end{array}$} \\
\hline$C D K N 1 A$ & Cyclin-dependent kinase inhibitor 1 & & \\
\hline CLDN1 & Claudin 1 & PTGIR & Prostaglandin 12 (prostacyclin) receptor \\
\hline CLDN5 & Claudin 5 & PTGIS & Prostaglandin 12 (prostacyclin) synthase \\
\hline \multirow[t]{2}{*}{ CTNNB1 } & Catenin (cadherin-associated protein), & PTGS1 & Prostaglandin-endoperoxide synthase 1 \\
\hline & beta $1,88 \mathrm{kDa}$ & PTGS2 & Prostaglandin-endoperoxide synthase 2 \\
\hline CTNNBIP1 & Catenin, beta interacting protein 1 & PTK2 & Protein tyrosine kinase 2 \\
\hline DUSP1 & Dual specificity phosphatase 1 & \multirow[t]{2}{*}{$R A C 1$} & \multirow{2}{*}{$\begin{array}{l}\text { Ras-related C3 botulinum toxin } \\
\text { substrate } 1\end{array}$} \\
\hline ECE1 & Endothelin converting enzyme 1 & & \\
\hline EDN1 & Endothelin 1 & $R A P 1 A$ & Ras-related protein Rap-1A \\
\hline$E D N 2$ & Endothelin 2 & $R D X$ & Radixin \\
\hline ELAVA1 & $\begin{array}{l}\text { ELAV (embryonic lethal, abnormal vision, } \\
\text { Drosophila)-like } 1 \text { (Hu antigen } \mathrm{R} \text { ) }\end{array}$ & $R E L A$ & $\begin{array}{l}\text { v-rel reticuloendotheliosis viral oncogene } \\
\text { homolog } A\end{array}$ \\
\hline ESR1 & Estrogen receptor 1 & $R H O A$ & Ras homolog family member $\mathrm{A}$ \\
\hline$E Z R$ & Ezrin & ROCK1 & Rho-associated, coiled-coil containing protein \\
\hline F11R & F11 receptor & & kinase 1 \\
\hline FLT1 & Fms-related tyrosine kinase 1 & S100A3 & S100 calcium binding protein A3 \\
\hline$G A B P$ & GA binding protein transcription factor & SELE & Selectin E \\
\hline GAPDH & Glyceraldehyde-3-phosphate dehydrogenase & SERPINE1 & Serpin peptidase inhibitor, clade E 1 \\
\hline GJA4 & Gap junction protein, alpha $4,37 \mathrm{kDa}$ & SOD1 & Superoxide dismutase 1 \\
\hline GPX1 & Glutathione peroxidase 1 & TRAF1 & TNF receptor-associated factor 1 \\
\hline HMOX1 & Heme oxygenase (decycling) 1 & VCAM1 & Vascular cell adhesion molecule 1 \\
\hline HSBP1 & Heat shock factor binding protein 1 & VEGFA & Vascular endothelial growth factor A \\
\hline HSP9O & Heat shock protein 90 & $V W F$ & von Willebrand factor \\
\hline
\end{tabular}

Appendix 1. Continued
IL6

IQGAP1

IQGAP2

ITGB1

JAM3

JUN

$K D R$

KEAP1

$K L F 2$

LDLR

MAPK14

MSN

MYL12B

MYL7

MYLK

NFKB1

NOS3

NOX1

NOX3

NOX4

NPR1
Inhibitor of kappa light polypeptide gene

enhancer in B-cells, kinase beta

Interleukin 6

IQ motif containing GTPase

activating protein 1

IQ motif containing GTPase

activating protein 2

Integrin, beta 1

Junctional adhesion molecule 3

Jun proto-oncogene

Kinase insert domain receptor

Kelch-like $\mathrm{ECH}$-associated protein 1

Kruppel-like factor 2

Low density lipoprotein receptor

Mitogen-activated protein kinase 14

Moesin

Myosin, light chain 12B

Myosin, light chain 7

Myosin light chain kinase

Nuclear factor of kappa light polypeptide gene enhancer in B-cells 1

Nitric oxide synthase 3

NADPH oxidase 1

NADPH oxidase 3

NADPH oxidase 4

Natriuretic peptide receptor A/guanylate cyclase A 